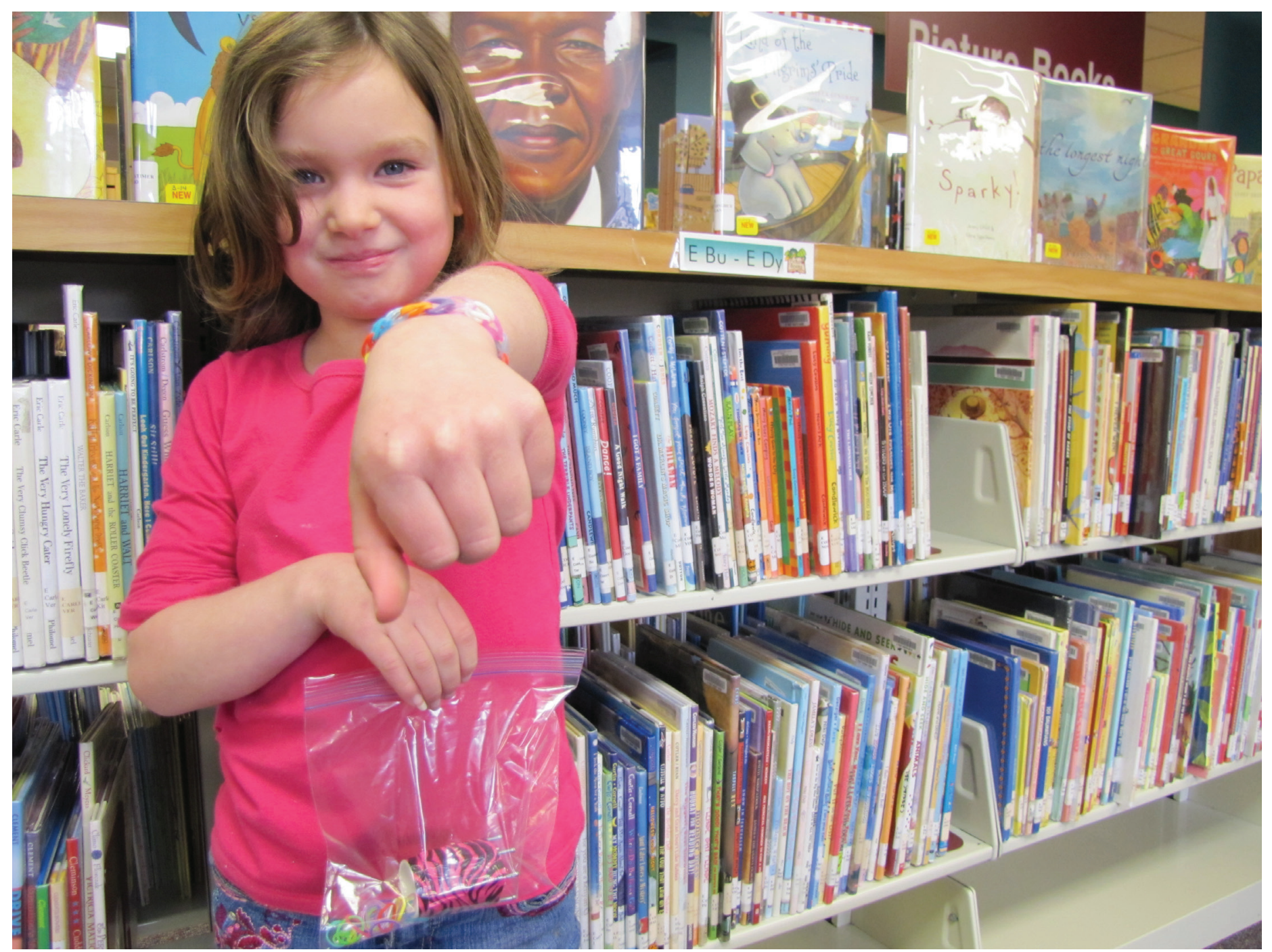

Isabella shows off her bracelet while holding her takeaway kit.

\title{
Maker \\ Program: Loom Lab
}

Sarah Cournoyer

W

hile my library does not have a dedicated makerspace, I look for every opportunity to offer maker programs and Loom Lab is one of these.

Loom bracelets, which are currently a hot item, make an ideal library program. Yet, when I started looking into it, the cost of the looms themselves gave me pause. Did I want to allocate programming dollars to something that might be a brief fad?

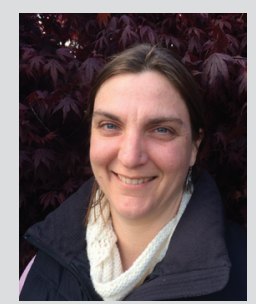

Sarah Cournoyer is Assistant Director and Children's Librarian at Horicon (WI) Public Library. 


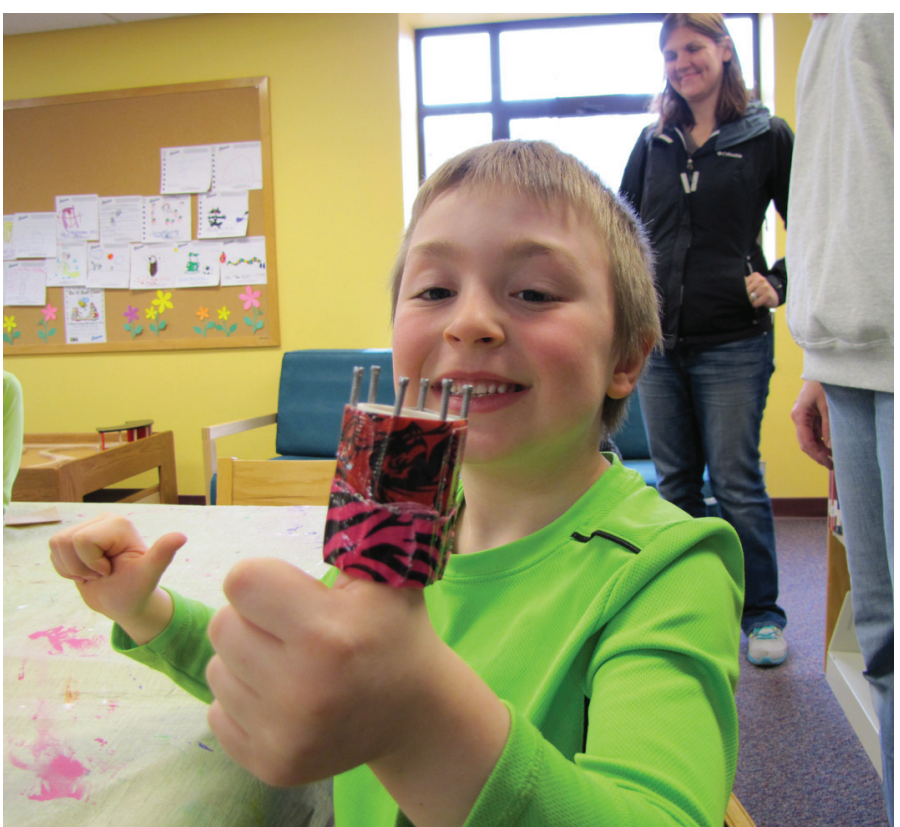

Whose loom gets two thumps up? This guy's!

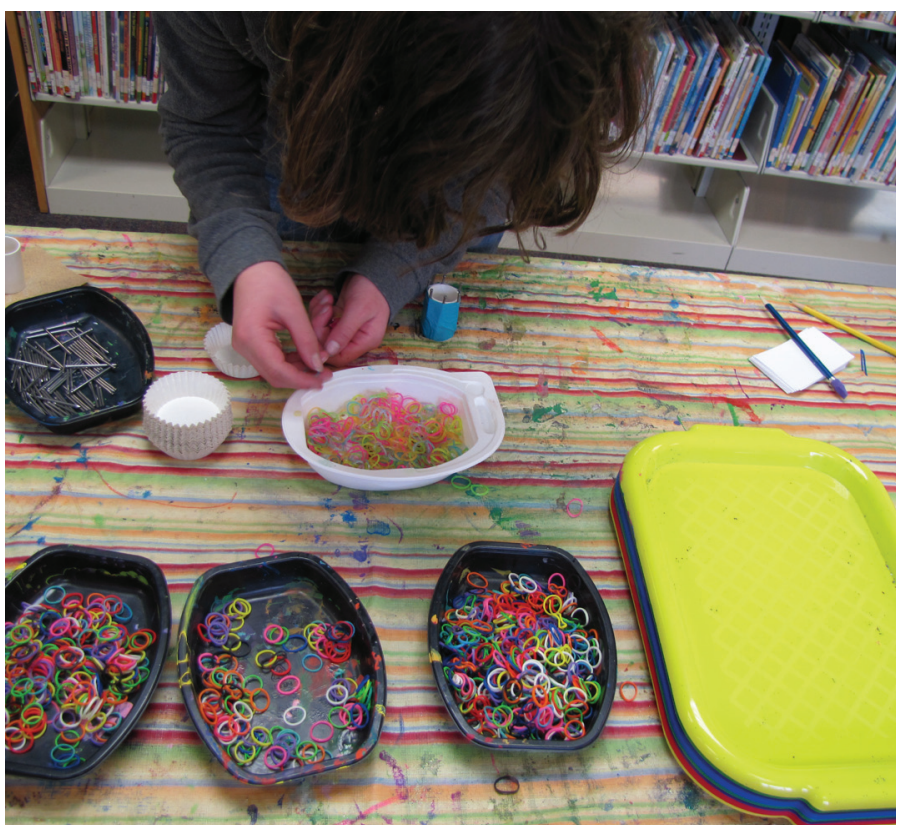

Bracelet making encourages sorting and pattern making.

Then a colleague mentioned that she had made looms at her library with items already in the supply closet. What's better than a loom bracelet? Making a loom to make loom jewelry, of course! The Loom Lab was born.

The necessary supplies are very simple:

- PVC pipe cut into 2-inch lengths (I used 1.5-inch diameter pipe)

- medium-grade sandpaper

- six finishing nails per loom

- duct tape

At no cost to us, the local hardware store cut the PVC pipe into the right lengths; our costs were $\$ 3.59$ for a 10 -foot piece, which made sixty looms.

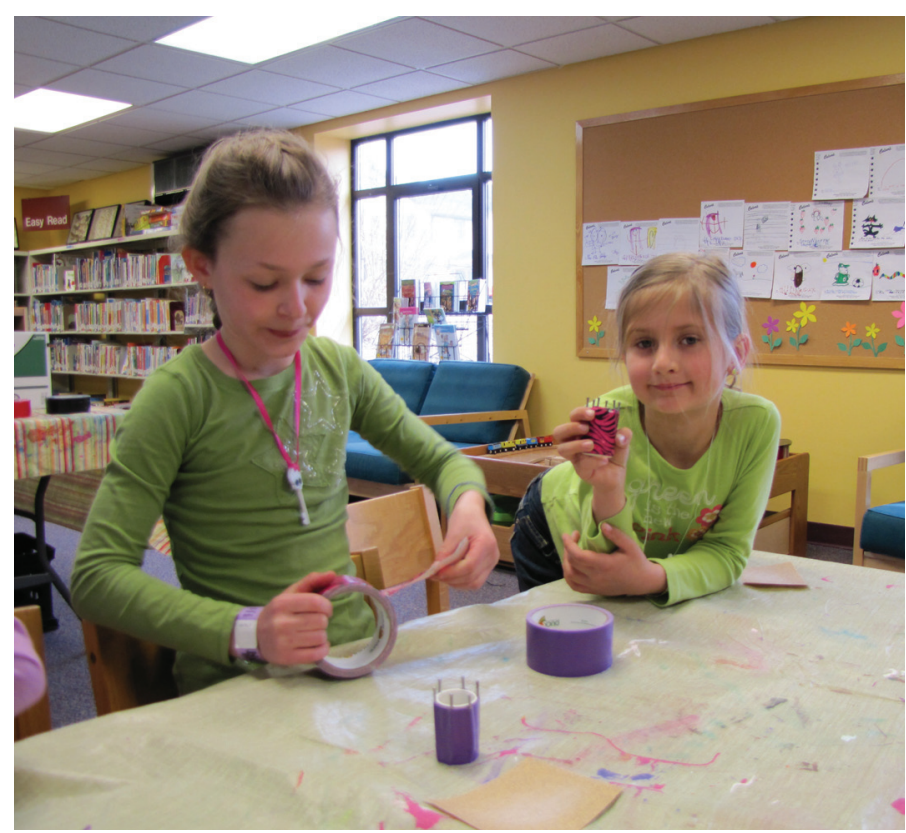

Once it's wrapped, the loom is ready to go.

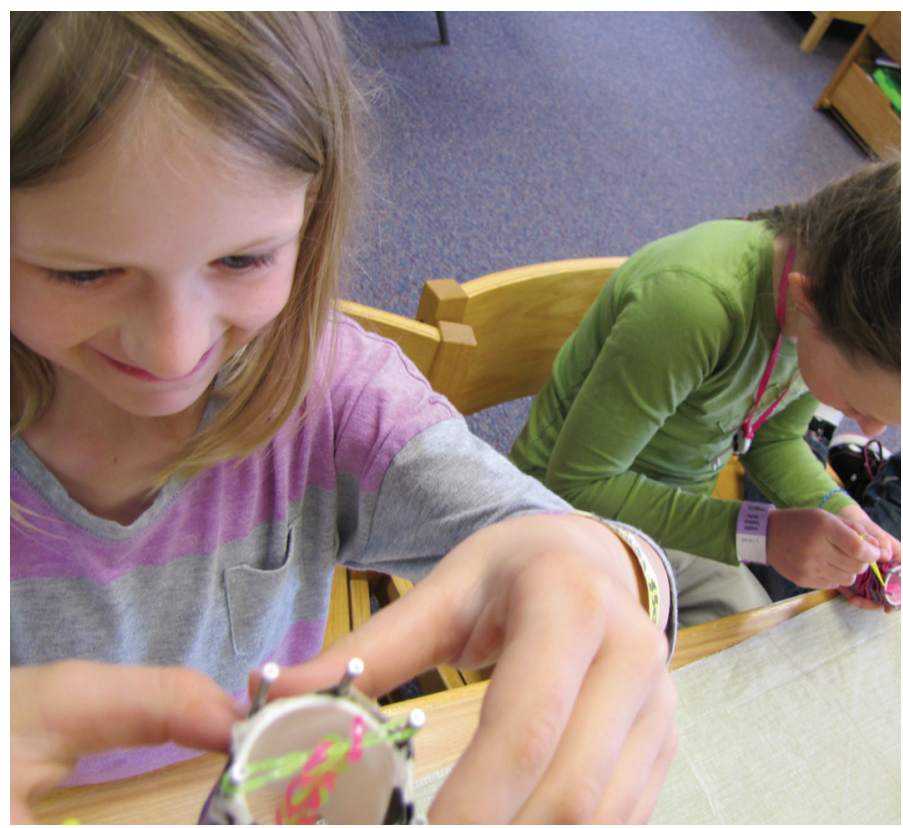

Pleased with her progress.

The expensive part was the duct tape, at $\$ 4$ or more per roll, but if you already have some in your supply closet, you're good to go. One loom uses less than twelve inches of duct tape.

I also purchased several packs of elastic bands, including glowin-the-dark ones, from a local craft store. A package with five hundred bands and twenty-five closures cost $\$ 3.99$. Because a simple two-band, two-loop bracelet only takes twenty to thirty bands, these packages go a long way. However, more advanced bracelets, like the fishtail, use more bands.

Children as young as three years old made these pieces. Many of the thirty children who came to the program already knew how to use a different style of loom, and several of them were happy to show me how to make a bracelet using nothing but their own two fingers! I learned something, too! \&. 